\title{
THE WILLMORE CONJECTURE IN THE REAL PROJECTIVE SPACE
}

\author{
Antonio Ros
}

\begin{abstract}
We prove that for any torus $M$ immersed in the real projective space $\mathbb{R P}^{3}(1)$ with mean curvature $H$, we have that $\int_{M}\left(1+H^{2}\right) d A \geq \pi^{2}$ and that the equality holds only for the minimal Clifford torus. In terms of the three sphere, this result says that the Willmore conjecture is true for immersed tori in $S^{3}(1)$ invariant under the antipodal map.
\end{abstract}

\section{Introduction.}

In 1965 Willmore, [13], conjectured that any torus $M$ immersed in the Euclidean space $\mathbb{R}^{3}$ satisfies the inequality

$$
\int_{M} H^{2} d A \geq 2 \pi^{2}
$$

$H$ being the mean curvature of $M$. The equality is attained for any conformal image (via the stereographic projection) of the minimal Clifford torus in the unit three-sphere $S^{3}(1)$. Although the general case remains unsolved, we have several partial answers to this conjecture. It is known to be true if $M$ is either a tube around an immersed closed curve, see Shiohama and Takagi [11] or Willmore [14], or the conformal image of a flat torus in $S^{3}(1)$, see Chen [2], or a torus of revolution, see Langer and Singer [5]. An important partial result was found by $\mathrm{Li}$ and Yau [7] who solve affirmatively the problem when the conformal structure induced by the immersion belongs to a certain bounded domain (described explicitly) of the moduli space of conformal structures on the torus. Montiel and Ros [8] give a larger domain in this moduli space for which the conjecture is true. Simon [12] proved the existence of a torus in $\mathbb{R}^{3}$ which minimizes the functional $\int_{M} H^{2} d A$. This torus is known to be embedded, see Li and Yau [7], and unknotted, see Langevin and Rosenberg [6]. Other related results can be found in Bryant [1], Kuhnel and Pinkall [3] and Kusner [4].

The functional above is well-known to be invariant under conformal transformations and the Willmore conjecture, when rewritten in terms of spherical surfaces, says that any torus $M$ immersed in the unit sphere $S^{3}(1)$ verifies $\int_{M}\left(1+H^{2}\right) d A \geq 2 \pi^{2}, H$ being the mean curvature of $M$ in $S^{3}(1)$, and that the

Received August 17, 1999.

Mathematics Subject Classification. 53A10, 53A05, 53C42.

Research partially supported by a DGYCYT Grant No. PB94-0796. 
equality holds only for the minimally embedded Clifford torus and its images by conformal transformations of the sphere.

In this paper we solve the Willmore conjecture for tori in the sphere which are invariant under the antipodal map or, equivalently, we find the minimum of the Willmore functional for tori immersed in the three dimensional real projective space $\mathbb{R P}^{3}(1)$. More precisely, we prove that:

If $M$ is a torus immersed in $\mathbb{R P}^{3}(1)$, then $\int_{M}\left(1+H^{2}\right) d A \geq \pi^{2}$. If the equality holds, then $M$ is the (projection to the projective space of the) minimal Clifford torus.

In fact we show that the above inequality holds for any orientable surface immersed in the projective space. In terms of spherical surfaces our result say that:

If $M$ is an immersed torus in the sphere $S^{3}(1)$ invariant under the antipodal map, then $\int_{M}\left(1+H^{2}\right) d A \geq 2 \pi^{2}$ and the equality holds if and only if $M$ is the minimal Clifford torus.

The same result is true if $M$ is not a torus but any compact orientable surface of odd genus.

The main ingredient in our proof is the solution of the isoperimetric problem in the projective space $\mathbb{R P}^{3}(1)$ given by Ritoré and Ros in [9].

\section{Preliminaries.}

We shall need the following result

Theorem 1. If $M$ is a compact surface immersed in $S^{3}(1)$, then

$$
\int_{M}\left(1+H^{2}\right) d A \geq 4 \pi,
$$

and the equality holds if and only if $M$ is an umbilic sphere. Moreover, if $M$ is not embedded, then

$$
\int_{M}\left(1+H^{2}\right) d A>8 \pi .
$$

The first part of the theorem is the result of Willmore [13] transported to the sphere via stereographic projection. It can be obtained from a lot of different arguments. We propose our own proof in Remark 1. The second statement is proved by Li and Yau [7] (see also the comments in [9] for the strict inequality).

Given a closed subset $\Omega$ in $S^{3}(1)$ or in $\mathbb{R P}^{3}$ we will denote its volume by $V(\Omega)$ and the area of its boundary, when it makes sense, by $A(\partial \Omega)$. The isoperimetric problem consists of finding among the subsets of given volume the ones which have the smallest area at its boundary. In the sphere it is well-known that the solutions of this problem are the geodesic balls. In the three dimensional projective space the isoperimetric problem was solved by Ritoré and Ros, [9], who proved that the solutions are the geodesic balls, solid tubes around geodesics or the complementary domains of geodesic balls, depending of the value of the 
fixed volume. This fundamental problem is unsolved for the other elliptic space forms, even for lens spaces, and the same holds for other simple compact 3manifolds like flat three tori, see the final remark of [10] for some results in this direction.

The following is a particular case of the theorem in [9]. It asserts that the solution of the isoperimetric problem for domains whose volume is one half of the volume of $\mathbb{R P}^{3}(1)$ is the domain enclosed by the embedded minimal Clifford torus, i.e., the projection to the projective space of the minimal Clifford torus in the 3-sphere. It will be convenient for us to state the result in terms of antipodal invariant domains in the three sphere.

Theorem 2. Let $\Omega \subset S^{3}(1)$ be a closed subset invariant under the antipodal map with $V(\Omega)=\frac{1}{2} V\left(S^{3}(1)\right)$ and whose boundary has well defined area. Then

$$
A(\partial \Omega) \geq 2 \pi^{2} \text {. }
$$

Moreover, if $\partial \Omega$ is a smooth surface, then the equality in (3) holds if and only if $\partial \Omega$ is the minimally embedded Clifford torus.

\section{The theorem.}

Let $\psi: M \rightarrow S^{3}(1) \subset \mathbb{R}^{4}$ be an oriented surface immersed in the unit threesphere and $N: M \rightarrow S^{3}(1)$ its unit normal vector field. Consider, for any $t \in \mathbb{R}$, the parallel map $\psi_{t}: M \rightarrow S^{3}(1)$ defined as $\psi_{t}=\cos t \psi+\sin t N$. For each $p \in M$, take a positive orthonormal basis $e_{1}, e_{2} \in T_{p} M$ of principal directions with principal curvatures $k_{1}$ and $k_{2}$ respectively. The differential of $\psi_{t}$ at $p$ is given by $\left(\psi_{t}\right)_{*}\left(e_{i}\right)=\left(\cos t-k_{i} \sin t\right) e_{i}, i=1,2$. So we get that the Jacobian of $\psi_{t}$ is $\left(\operatorname{Jac} \psi_{t}\right)(p)=\left(\cos t-k_{1} \sin t\right)\left(\cos t-k_{2} \sin t\right)$.

Lemma 1. Given $t, 0 \leq t<\pi$, the Jacobian of the map $\psi_{t}$ parallel to $\psi$ verifies

$$
\operatorname{Jac} \psi_{t} \leq 1+H^{2}
$$

$H=\frac{1}{2}\left(k_{1}+k_{2}\right)$ being the mean curvature of immersion $\psi$. Moreover, the equality holds everywhere on $M$ (assumed to be connected) if and only if either $\psi$ is totally umbilical and $\psi_{t}$ is totally geodesic, or $\psi$ is a minimal immersion and $t=0$.

Proof. We have that

$\operatorname{Jac} \psi_{t}=\cos ^{2} t-2 H \cos t \sin t+k_{1} k_{2} \sin ^{2} t \leq \cos ^{2} t-2 H \cos t \sin t+H^{2} \sin ^{2} t$, with the equality holding if and only if either $t=0$ or the point is umbilical. Thus

$$
\operatorname{Jac} \psi_{t} \leq(\cos t-H \sin t)^{2} \leq\left(\cos ^{2} t+\sin ^{2} t\right)\left(1+H^{2}\right)=1+H^{2},
$$

where we have applied the Schwarz inequality to the vectors $(\cos t, \sin t)$ and $(1,-H)$.

If we have the equality everywhere on $M$ we obtain that either $t=0$ and the above vectors are proportional (which means that $M$ is minimal) or $0<t$ and 
$M$ is totally umbilical. In that case $M$ and $\psi_{t}(M)$ are isometric to 2-spheres $S, S_{t} \subset S^{3}(1)$ of radius $r$ and $r_{t}$ respectively and $\psi_{t}: S \rightarrow S_{t}$ is an homothety. Clearly Jac $\psi_{t}$ attains its maximum just for $r_{t}=1$. This proves the lemma.

Let $\Omega$ be one of the domains enclosed by a given closed surface $M$ embedded in $S^{3}(1)$. For any number $t, 0 \leq t<\pi$, we can consider the subset $B(\Omega, t)=$ $\left\{p \in S^{3}(1) / \operatorname{dist}(p, \Omega) \leq t\right\}$ of points whose (spherical) distance to $\Omega$ is less than or equal to $t$. The distance between $\Omega$ and a point in $B(\Omega, t)-\Omega$ is attained by a minimizing geodesic which meets the surface $M$ orthogonally. This distance is controlled by the cut function, $c: M \rightarrow\{0 \leq t<\pi\}$. If $N$ denotes the outward pointing unit normal along $M$ and $p \in M, c(p)$ is the last positive time, such that the normal geodesic $t \mapsto \cos t p+\sin t N(p)$ attains the distance to $M$. Thus, we have $B(\Omega, t)=\Omega \cup\{\cos s p+\sin s N(p) \mid p \in M, 0 \leq s \leq \min \{t, c(p)\}\}$. Moreover $c(p)$ is less than or equal to the first focal value along the normal geodesic at $p$ and, so, for any $t, 0 \leq t \leq c(p)$, the Jacobian at $p$ of the parallel surface $\psi_{t}$ is nonnegative, $\left(\operatorname{Jac} \psi_{t}\right)(p) \geq 0$. As any point of the boundary of $B(\Omega, t)$ lies at distance $t$ from $M$ we obtain that

$$
\partial B(\Omega, t) \subset \psi_{t}\left(\left\{p \in M \mid\left(\operatorname{Jac} \psi_{t}\right)(p) \geq 0\right\}\right) .
$$

Proposition 1. Let $M \subset S^{3}(1)$ be a compact surface embedded in the unit three-sphere and $\Omega \subset S^{3}(1)$ the closure of one of the components of $S^{3}(1)-M$. Then, for any $t, 0 \leq t<\pi$, we have

$$
A(\partial B(\Omega, t)) \leq \int_{M}\left(1+H^{2}\right) d A
$$

$H$ being the mean curvature of $M$. Moreover, the equality holds if and only if either $M$ is an umbilical surface and $B(\Omega, t)$ is a halfsphere in $S^{3}(1)$, or $M$ is a minimal surface and $t=0$.

Proof. From the inclusion (4) we have that

$$
A(\partial B(\Omega, t)) \leq A\left(\psi_{t}\left(\left\{p \in M \mid\left(\operatorname{Jac} \psi_{t}\right)(p) \geq 0\right\}\right)\right) \leq \int_{\left\{\operatorname{Jac} \psi_{t} \geq 0\right\}} \operatorname{Jac} \psi_{t} d A
$$

which, combined with Lemma 1 gives

$$
A(\partial B(\Omega, t)) \leq \int_{\left\{\operatorname{Jac} \psi_{t} \geq 0\right\}}\left(1+H^{2}\right) d A \leq \int_{M}\left(1+H^{2}\right) d A
$$

This proves the claimed inequality. If the equality holds then we conclude that Jac $\psi_{t} \geq 0$ on $M$ and, using again Lemma 1 we get that either $M$ is an umbilical sphere and $\psi_{t}$ is totally geodesic or $t=0$ and $M$ is a minimal surface.

Theorem 3. Let $\psi: M \rightarrow \mathbb{R P}^{3}(1)$ by a compact orientable surface immersed in the real projective space with mean curvature $H$. Then

$$
\int_{M}\left(1+H^{2}\right) d A \geq \pi^{2}
$$

and the equality holds if and only if $M$ is the minimally embedded Clifford torus. 
Proof. If the immersion $\psi$ lifts to an immersion of $M$ in the sphere we conclude directly from (1) that $\int_{M}\left(1+H^{2}\right) d A \geq 4 \pi$, which is more than we need.

If the above lift does not exist, then we can construct a two fold covering $\widetilde{M} \rightarrow M$ and an immersion $\widetilde{\psi}: \widetilde{M} \rightarrow S^{3}(1)$ locally congruent to $\psi$. Therefore, we have $2 \int_{M}\left(1+H^{2}\right) d A=\int_{\widetilde{M}}\left(1+H^{2}\right) d A$, where we denote again by $H$ and $d A$ the mean curvature of $\widetilde{\psi}$ and the measure of $\widetilde{M}$. If $\widetilde{\psi}$ is not an embedding, by using (2), we obtain that $\int_{M}\left(1+H^{2}\right) d A>4 \pi$.

Thus we can assume that $\widetilde{M}$ is connected, embedded in $S^{3}(1)$ and invariant under the antipodal map. Let $\Omega$ be the closure of the component of $S^{3}(1)-\widetilde{M}$ which has the smallest volume. As $M$ is orientable it follows that $\Omega$ is invariant under the antipodal map and, so, $B(\Omega, t)$ is also antipodally symmetric. As the volume of $B(\Omega, t)$ is a continuous function of $t$ and $V(\Omega) \leq \frac{1}{2} V\left(S^{3}(1)\right)$, we can find $t, 0 \leq t<\pi$ such that $V(B(\Omega, t))=\frac{1}{2} V\left(S^{3}(1)\right)$. By combination of $(5)$ and (3) we conclude that $\int_{\mathbb{M}}\left(1+H^{2}\right) d A \geq 2 \pi^{2}$. If the equality holds, then Lemma 1 implies that either $\widetilde{M}$ is an umbilical sphere, or $t=0$ and $\widetilde{M}$ is minimal. The first option is discarded because $\Omega$ must be antipodal invariant and so, we obtain from the equality case in Theorem 2 that $\widetilde{M}$ is the embedded Clifford torus in $S^{3}(1)$. This proves the theorem.

Remark 1. If $M$ is a compact surface embedded in the three sphere (non necessarily antipodal invariant), we can repeat the arguments above by changing the isoperimetric inequality in $\mathbb{R P}^{3}(1)$ by the one in $S^{3}(1)$. In this way we obtain another proof of the first result in Theorem 1.

Corollary 1. Let $M$ be a compact orientable surface of odd genus immersed in $S^{3}(1)$. If $M$ is invariant under the antipodal map, then

$$
\int_{M}\left(1+H^{2}\right) d A \geq 2 \pi^{2}
$$

and the equality holds if and only if $M$ is the embedded minimal Clifford torus.

Proof. As in the proof above we reduce to the case in which $M$ is embedded. If the antipodal map preserves the components of $S^{3}(1)-M$ we have that $M$ induces an orientable surface in $\mathbb{R P}^{3}(1)$ and so the result follows from Theorem 3 . If the antipodal maps reverses the orientation of $M$, then the induced surface in the projective space will be an embedded Klein bottle with handles. As Proposition 2 below says that this case cannot hold, we have proved the corollary.

In our last result we sumarize all the information we have for the Willmore functional in the real projective space.

Corollary 2. Let $M$ be a compact surface immersed in $\mathbb{R P}^{3}(1)$.

If $M$ is a sphere, then

$$
\int_{M}\left(1+H^{2}\right) d A \geq 4 \pi
$$


and the equality holds if and only if $M$ is an umbilical sphere.

If $M$ is an sphere with $n$ handles, $n \geq 1$, then

$$
\int_{M}\left(1+H^{2}\right) d A \geq \pi^{2},
$$

and the equality holds if and only if $M$ the minimal Clifford torus.

If $M$ is a projective plane with handles, then

$$
\int_{M}\left(1+H^{2}\right) d A \geq 2 \pi,
$$

and the equality holds if and only if $M$ is a linear plane.

If $M$ is a Klein bottle with handles, then

$$
\int_{M}\left(1+H^{2}\right) d A>4 \pi .
$$

In the first case the immersion lifts to $S^{3}(1)$ and the result follows from (1). The second case is our main result. The third one is simply the inequality (1) for antipodal invariant surfaces and the last one follows from Proposition 2 and (2).

Finally we remark that the inequalities above transform into area estimates in the particular (but interesting) case that $M$ is a minimal surface.

\section{Appendix.}

Any nonorientable compact surface is constructed by adding $n$ handles, $n=0,1,2, \ldots$, either to a projective plane or to a Klein bottle. Adding handles to a linear subvariety of $\mathbb{R P}^{3}$ we conclude that any surface of the first type can be embedded in the projective space. The next result shows that this is not possible for surfaces of the second type. This fact could be well known, but I have not found any reference about it. In any case, the proof below may be useful to some readers.

Proposition 2. A Klein bottle with handles cannot be embedded in the projective space $\mathbb{R P}^{3}$.

Proof. Suppose that such a surface exists and let $M \subset S^{3}(1)$ be its pullback image in the three sphere. If $M$ where nonconnected, then its components would be compact nonorientable surfaces embedded in the three sphere. As this is impossible we get that $M$ is a connected compact orientable surface invariant under the antipodal map $f: S^{3} \rightarrow S^{3}$. Thus $M$ covers twice the given Klein bottle with handles and in particular the genus of $M$ is odd. Moreover, as $f$ reverses the orientation of $M$, if we denote the components of $S^{3}(1)-M$ by $W_{1}$ and $W_{2}$ we have that $f\left(W_{1}\right)=W_{2}$ and $f\left(W_{2}\right)=W_{1}$.

Take and 2-dimensional equator $S$ in the three sphere which cuts $M$ transversally and denote the components of $S^{3}(1)-S$ by $S^{+}$and $S^{-}$. Because of the symmetry of $M$, the domains $M^{+}=M \cap S^{+}$and $M^{-}=M \cap S^{-}$are homeomorphic compact surfaces with the same boundary $\Gamma=M \cap S$ and $\Gamma$ consists 
in a disjoint union of $r$ Jordan curves. If $M^{+}$has $k$ connected components, $\Sigma_{1}, \ldots, \Sigma_{k}$, and each $\Sigma_{i}$ has genus $g_{i}$ and $r_{i}$ boundary components, we have that $r=r_{1}+\cdots+r_{k}$ and the Euler characteristic of $M$ can be computed as follows

$$
\begin{aligned}
\chi(M)=\chi\left(M_{+}\right)+\chi\left(M_{-}\right)= & 2 \chi\left(M_{+}\right)=2\left(\chi\left(\Sigma_{1}\right)+\cdots+\chi\left(\Sigma_{k}\right)\right)= \\
2\left(2-2 g_{1}-r_{1}+\cdots+2-2 g_{k}-r_{k}\right) & =4 m-2 r,
\end{aligned}
$$

for some integer number $m$. As the genus of $M$ is odd we conclude that $r$ is even.

On the other hand, as the antipodal map $f$ preserves $S-\Gamma=\left(W_{1} \cup W_{2}\right) \cap S$ but does not preserve any of its components (because $f$ interchanges $W_{1}$ and $W_{2}$ ) we obtain that the number of connected components of $S-\Gamma$ is even. As this contradicts the assertion below (which is obtained by a repeated application of the Jordan curve theorem) we conclude that $M$ cannot exist, as we claimed.

Assertion. If $\Gamma \subset S$ is the union of $r$ pairwise disjoint Jordan curves, then $S-\Gamma$ has exactly $r+1$ connected components.

\section{References}

[1] R. Bryant, A duality theorem for Willmore surfaces, J. Diff. Geom. 20 (1984) 23-53.

[2] B.Y. Chen, On the total curvature of immersed manifolds $V$. C-surfaces in Euclidean m-space, Bull. Inst. Math. Acad. Sinica 9 (1981) 509-516.

[3] W. Kuhnel and U. Pinkall, On total mean curvatures, Quart. J. Math. Oxford Ser. (2) 37 (1986) 437-447.

[4] R. Kusner, Comparison surfaces for the Willmore problem, Pacific J. Math. 138 (1989) $317-345$.

[5] J. Langer and D. Singer, Curves in the hyperbolic plane and mean curvature of tori in 3-space, Bull. London Math. Soc. 16 (1984) 531-534.

[6] R. Langevin and H. Rosenberg, On curvature integrals and knots, Topology 15 (1976) 405-416.

[7] P. Li and S.-T. Yau, A new conformal invariant and its applications to the Willmore conjecture and first eigenvalue of compact surfaces, Invent. Math. 69(1982) 269-291.

[8] S. Montiel and A. Ros, Minimal immersions of surfaces by the first eigenfunctions and conformal area, Invent. Math. 83 (1986) 153-166.

[9] M. Ritoré and A. Ros, Stable constant mean curvature tori and the isoperimetric problem in three space forms, Comment. Math. Helv. 67 (1992) 293-305.

[10] _ The spaces of index one minimal surfaces and stable constant mean curvature surfaces embedded in flat three manifolds, Trans. Amer. Math. Soc. 348 (1996) 391-410.

[11] K. Shiohama and R. Takagi, A characterization of a standard torus in $E^{3}$, J. Diff. Geom. 4 (1970) 477-485.

[12] L. Simon, Existence of surfaces minimizing the Willmore functional, Comm. Anal. Geom. 2 (1993) 281-326.

[13] T.J. Willmore, Note on embedded surfaces, An. Sti. Univ. "Al. I. Cuza" Iasi Sect. I a Mat. 11 (1965) 493-496.

[14] _ Mean curvature of Riemannian immersions, J. London Math. Soc. (2) 3 (1971) $307-310$. 
Departamento de Geometria y Topologia, Facultad de Ciencias - Universidad de Granada, Fuentenueva, 18071 - Granada - SPAin

E-mail address: aros@@goliat.ugr.es 